\title{
ZNF384 rearrangement in acute lymphocytic leukemia with renal involvement as the first manifestation is associated with a poor prognosis: a case report
}

\author{
Jinlong $\mathrm{Ma}^{\dagger}$, Jiaheng Guan ${ }^{\dagger}$ and Baoan Chen ${ }^{*}$
}

\begin{abstract}
Background: Novel fusion genes such as ZNF384, have been identified in B-cell precursor acute lymphoblastic leukemia (BCP-ALL) in recent years. Patients harboring ZNF384 rearrangements have a distinctive immunophenotype with weak CD10 and aberrant CD13 and/or CD33 expression. Thus, ZNF384-rearranged ALL is a unique subtype of BCP-ALL. However, research on the prognostic significance of ZNF384 rearrangements has been limited to date, especially in adolescents.

Case presentation: We described a 17-year-old adolescent who was diagnosed with ALL and had renal involvement as the first manifestation, which was very rare in the existing studies. FISH analysis indicated a rearrangement of ZNF384 according to its probe. The patient had a typical characteristic immunophenotype of ZNF384 rearrangement, with CD10 negativity and CD13 and CD33 positivity. She had an unfavorable prognosis because she responded poorly to chemotherapy and developed a relapse shortly after reaching $C R$.

Conclusion: The importance of ZNF384 rearrangements in terms of prognosis remains unclear. We reported an adolescent who was diagnosed with ZNF384-rearranged ALL with renal involvement. She underwent different therapies, but her prognosis remained poor. Since ZNF384 rearrangements may act as a prognostic predictor in children or adolescents, early detection based on its characteristic immunophenotype is of great necessity.
\end{abstract}

Keywords: Acute lymphocytic leukemia, ZNF384, Gene rearrangement, Immunophenotype, Prognosis

\section{Background}

Acute lymphocytic leukemia (ALL) is a malignant neoplasm in which the differentiation of lymphoid cells is blocked at an early stage, and there is extensive infiltration in the bone marrow, peripheral blood, and other organs. Over $80 \%$ of ALL cases are B-cell precursor acute lymphoblastic leukemia (BCP-ALL). In 2020, 6,150 new cases of ALL were diagnosed in the United States,

*Correspondence: cba8888@hotmail.com

†Jinlong Ma and Jiaheng Guan contributed equally to this article Department of Hematology and Oncology, School of Medicine, Zhongda Hospital, Southeast University, Nanjing 210009, Jiangsu, China accounting for $0.3 \%$ of all new cancer cases [1]. ALL usually occurs in children and young adults, with the peak age ranging from 2 to 5 [2].

Gene rearrangements and fusions are common in BCP-ALL, and they play an important role in determining therapeutic targets and predicting prognosis. With the advancement of genome and transcriptome sequencing, novel gene fusions, such as zinc-finger protein 384 (ZNF384) rearrangements have been found. The ZNF384 gene is located on chromosome $12 \mathrm{p} 13$. It encodes a zinc finger protein that functions as a transcription factor and regulates the expression of matrix metalloproteinases [3]. In ZNF384-rearranged BCP-ALL, the breakpoints original author(s) and the source, provide a link to the Creative Commons licence, and indicate if changes were made. The images or other third party material in this article are included in the article's Creative Commons licence, unless indicated otherwise in a credit line to the material. If material is not included in the article's Creative Commons licence and your intended use is not permitted by statutory regulation or exceeds the permitted use, you will need to obtain permission directly from the copyright holder. To view a copy of this licence, visit http://creativecommons.org/licenses/by/4.0/. The Creative Commons Public Domain Dedication waiver (http://creativecommons.org/publicdomain/zero/1.0/) applies to the data made available in this article, unless otherwise stated in a credit line to the data. 
of ZNF384 are typically located in exons 2 and 3, which contain the entire ZNF384 protein that may be responsible for the characteristics of the immunophenotype [4, 5]. Therefore, the presence of ZNF384 rearrangements should be a hallmark and the diagnostic criterion of a separate subtype of BCP-ALL since patients harboring such rearrangements have a distinctive immunophenotype. This immunophenotype includes weak CD10 and aberrant CD13 and/or CD33 expression (6).

Recent data have shown that as many as $1 \%$ to $6 \%$ of children and $5 \%$ to $15 \%$ of adults with BCP-ALL harbor ZNF384 rearrangements [7]. Although the incidence is not low, such rearrangements are difficult to detect by conventional karyotype analysis, and there has been a lack of research on the prognosis of this rearrangement for a long time. Furthermore, the liver, spleen, and lymph nodes are the most common sites of extramedullary involvement in ALL, with renal involvement being relatively uncommon. The unique immunophenotype of ZNF384 rearrangements helps to diagnose BCP-ALL patients with rare sites of extramedullary involvement. Herein, we report an adolescent case of ZNF384-rearranged ALL with renal involvement as the first manifestation and poor prognosis.

\section{Case presentation}

A 17-year-old female was admitted to our hospital in December 2019 because of increased urine foam and fatigue for 3 weeks.

Laboratory examinations showed protein $(3+)$ and occult blood $(2+)$ in urine. The blood urea nitrogen was $5.39 \mathrm{mmol} / \mathrm{l}$, the serum creatinine was $69 \mu \mathrm{mol} / \mathrm{l}$, and the lymphocyte ratio was $62.01 \%$. Kidney color Doppler ultrasound showed increased volume in both kidneys as well as enhanced parenchymal echo. Then kidney biopsy was performed, indicating lymphoblastic lymphoma or leukemia with kidney involvement (Fig. 1A and B). Immunohistochemistry showed $\operatorname{TdT}(+)$, CD99(+), CD3(-), CD20(part+), CD73(-), PAX5(+), and $\mathrm{LCA}($ part +$)$. Fluorescence in situ hybridization (FISH) of the kidney biopsy sample indicated ZNF384 rearrangement (Fig. 1E). Then, bone marrow aspiration and biopsy were performed, revealing the disappearance of fat vacuoles and the appearance of immature lymphoid cells. However, conventional G-banding cytogenetic analysis showed a normal bone marrow karyotype (Fig. 2). A suspected diagnosis of ALL was made. There was extreme lymphocyte proliferation and the proportion of lymphoblasts was $50.8 \%$ (Fig. $1 \mathrm{C}$ and D). Flow cytometry (FCM) of bone marrow revealed CD34(+), CD117(-), CD33(+), CD64(-), CD13(+), CD14(-), CD274(-), TSLPR(-), CD11b(-), $\operatorname{IgM}(+)$, CD71(-), CD56(+), CD2(-), CD7(-), CD5(-),
$\mathrm{CD} 10(+), \quad \mathrm{CD} 3(-), \quad \mathrm{CD} 4(-), \quad \mathrm{CD} 8(-), \quad \mathrm{CD} 38(+)$, CD81(+), HLA-DR(+), CD19(+), CD22(+), CD20(+), cMPO(-), cCD3(-), cCD79a $(+), \operatorname{TDT}(+), \operatorname{CD} 58(+)$, CD61(-), CD235a(-), and CD11C(-), and these findings are compatible with BCP-ALL (Fig. 3). Nextgeneration sequencing (NGS) showed STAG2 gene mutations in the bone marrow, and reverse transcription-polymerase chain reaction (RT-PCR) showed that common fusion genes for BCP-ALL, including TCF3PBX1, TCF3-HLF, ETV6-RUNX1 and BCR-ABL, were negative. Other less common fusion genes for Ph-like ALL were also negative.

Her medical history was unremarkable. On physical examination, the patient had an anemic appearance without ecchymosis. The initial laboratory evaluation revealed lymphocytosis $\left(2.37 \times 10^{9} / 1\right)$ and moderate anemia (Hb78 g/l, vitamin B12 $164 \mathrm{pg} / \mathrm{ml}$, folic acid $3.00 \mathrm{ng} / \mathrm{ml}$ and serum ferritin $426.7 \mathrm{ug} / \mathrm{l})$. She was diagnosed with BCP-ALL with involvement of both kidneys.

After a cycle of the VCDLP regimen, the bone marrow was obviously hyperplastic and active, and immature lymphocytes were occasionally observed. FCM showed the ratio of lymphoblasts was $42 \%$ with CD34(+), CD10(-), CD19(+), CD38(+), HLA-DR(+), CD64(-), CD13(+), CD20(-), and CD33(+). Since the patient did not achieve remission, a cycle of FLAG salvage treatment was administered, and then the patient was assessed as complete remission (CR). No lymphoblasts were seen in the bone marrow. FCM showed that the ratio of lymphoblasts was $0.8 \%$ with CD34(+), CD10(-), CD19(+), CD38(+), HLA-DR(+), CD13(+) and STAG2 gene mutations. The volume of both kidneys returned to normal according to color Doppler ultrasound. After the patient achieved CR, an intrathecal drug injection was performed for consolidation therapy. Eight months after the continued complete remission (CCR), the disease relapsed. A bone marrow smear revealed 55.2\% lymphoblasts, and FCM showed ALL with partial expression of CD33. FISH showed ZNF384 rearrangement (39\%) according to its probe (Fig. 1F) and positivity for IgH rearrangement (37\%). The leukemia fusion genes and mutation panels were both negative. However, there was no remission in the bone marrow after she was treated with chidamide and a dose-adjusted FLAG plus VP chemotherapeutic regimen, as well as a highly sensitive treatment, HAD. The percentage of lymphoblasts was $14.4 \%$ and ZNF384 rearrangement was positive (17.6\%) according to FISH. Currently, the patient has been admitted to the hospital and is receiving chemotherapy regularly. The details of the treatment process are summarized in Additional file 1. 

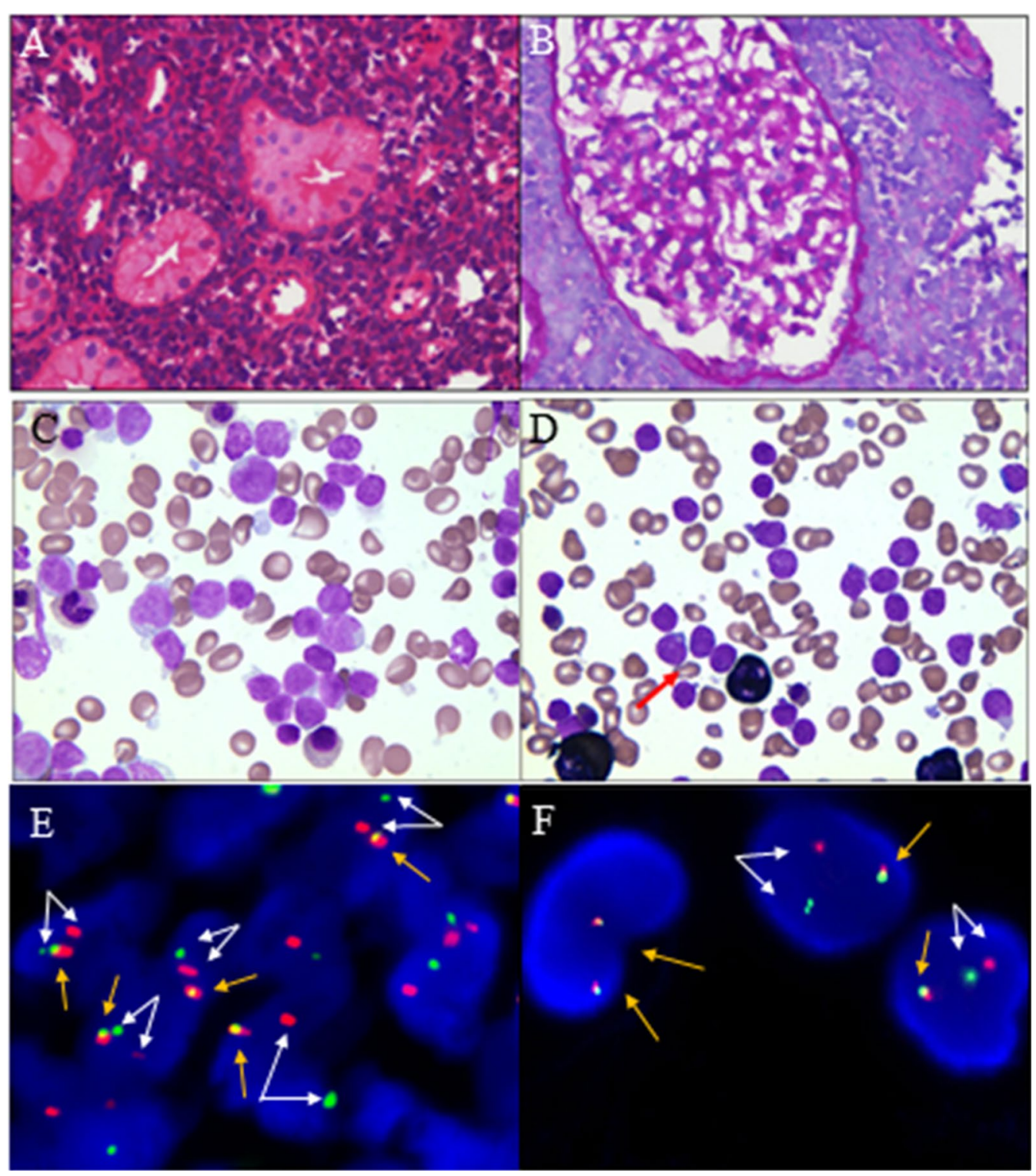

Fig. 1 Pathological results of kidney biopsy and bone marrow aspiration smear and results of FISH. A, B The result of kidney biopsy shows increased lymphocytes, indicating lymphoblastic lymphoma or leukemia with kidney involvement (A: HE staining; original magnification, $\times 400$ and $\mathbf{B}$ : PAS staining; original magnification, $\times 400)$. C The result of Wright-Giemsa staining of the bone marrow smear sample is shown. Bone marrow hyperplasia is extremely active, with lymphoblasts accounting for $50.8 \%$ of cells before treatment (Wright-Giemsa staining; original magnification, $\times 1000$ ). D Lymphoblasts were negative for myeloperoxidase (red arrow), suggesting the possible diagnosis of acute lymphocytic leukemia (POX staining; original magnification, $\times 1000$ ). E Fluorescence in situ hybridization (FISH) using a ZNF384 break-apart probe shows ZNF384 rearrangement in the kidney biopsy sample. Recognizable split signals can be seen in the scattered individual cells. The white arrows represent a single red signal and a single green signal (1R1G), which are split signals. The yellow arrow represents a single yellow signal (1Y), which is a normal signal. 1R1G1Y is a positive signal model for ZNF384 rearrangement. F Describes the ZNF384 rearrangement (39\%) in the bone marrow sample, indicating homology with the rearrangement of ZNF384 in the kidney biopsy sample. The two yellow arrows on the left represent two yellow signals, which are negative signals for ZNF384 rearrangement. The white arrows and the yellow arrows on the right are indicative of ZNF384 gene rearrangement (1R1G1Y) 


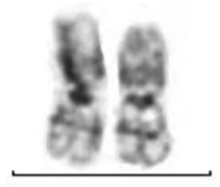

1
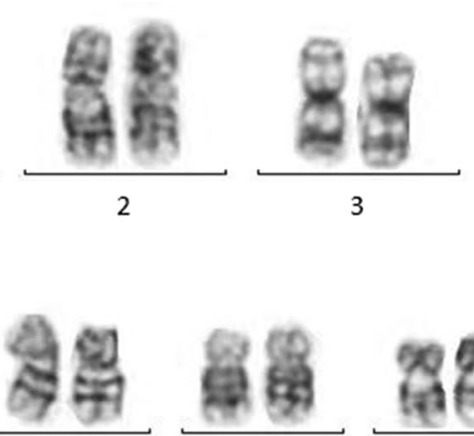

8

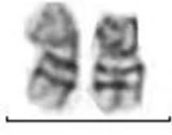

7

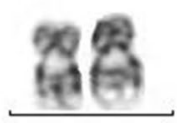

9

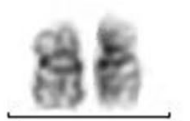

10

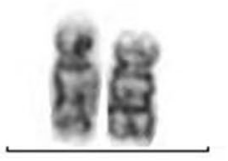

4

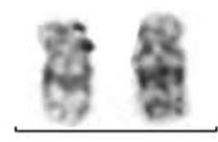

5

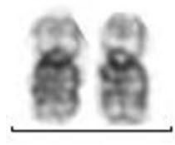

6

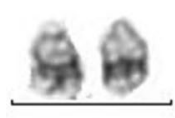

13

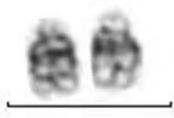

14

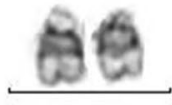

15

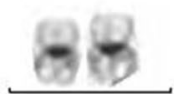

16

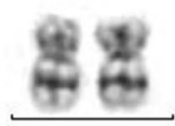

11

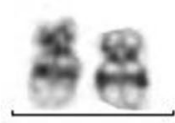

12

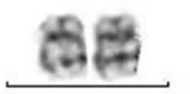

17

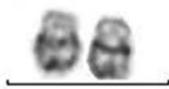

18
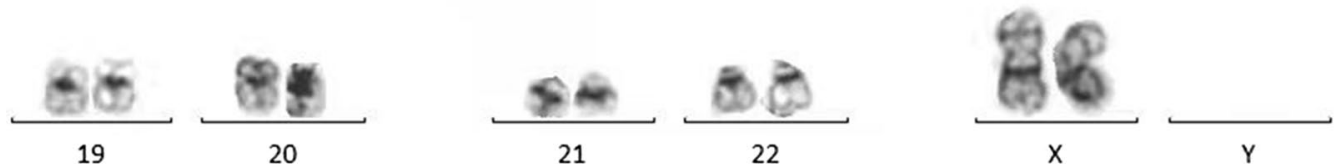

Fig. 2 Karyotype analysis of chromosomal G-banding. The G-banded karyotype of bone marrow cells showed a normal karyotype upon admission to our hospital

\section{Discussion and conclusions}

Patients harboring ZNF384 rearrangements are likely to possess the characteristic immunophenotype of a dull or negative CD10 and aberrant expression of one or more myeloid antigens. Thus, ZNF384-rearranged ALL is a new subtype of leukemia that may be diagnosed as BCPALL [8]. CD10 was negative in our case, while CD13 and CD33 were positive, which is consistent with previous studies. The Tokyo Children's Cancer Study Group (TCCSG) reported a similar outcome to ours. TCCSG tested 91 samples from BCP-ALL patients, revealing an incidence of a weak or negative expression of CD10, ranging from 0.39 to $67.34 \%$ (mean: $19.44 \pm 18.23 \%$ ), and $31.82 \%$ and $77.27 \%$ expression of CD13 and CD33, respectively, in 22 patients with ZNF384-related fusion genes [5].

Unlike the mechanism by which subclonal genomic variation drives clonal evolution in the disease progression of ALL [9], mutational variegation does not determine the immunophenotype for individual patients harboring ZNF384 rearrangements, and immunophenotypic aberrancy arises from inherent lineage plasticity [8]. Moreover, fusion partners for ZNF384 are commonly involved in BCP-ALL, which is responsible for leukemia development. Preclinical studies found that the initial effect of fusion proteins was the inhibition of B-cell differentiation by introducing EP300, one of the fusion genes, and ZNF384 into mouse pro-B cells, resulting in the stasis of cell differentiation $[10,11]$.

The prognosis of ZNF384 rearrangements in children or adolescents remains unclear. A retrospective study analyzed a total of 218 ZNF384-rearranged ALL cases, with ages ranging from 1 to 25 . The 5 -year event-free survival (EFS) rate was $85 \%$ (95\% CI 78-90\%), and the 5-year overall survival (OS) rate was 91\% (95\% CI 85-95\%) for all patients, suggesting a good outcome [12]. Mary Shago also analyzed a cohort of 240 pediatric patients who were diagnosed with BCP-ALL. Seven of the 240 patients were identified to have ZNF384 rearrangements, with an average age of 5.1 years. The EFS ranges from 6 years 2 months to 9 years 2 months and all patients achieved remission with no relapse [13]. Although a few studies support a generally favorable prognosis in children or young adults with ZNF384 rearrangements, others suggest a poor clinical outcome. A higher recurrence rate of over $2 \%$ was reported in some pediatric ZNF384 gene fusion cases [5]. Nishimura reported two pediatric TCF3ZNF384-positive patients who developed disease relapse more than 10 years after diagnosis [14]. Additionally, CD10 negativity was found to be an independent adverse 

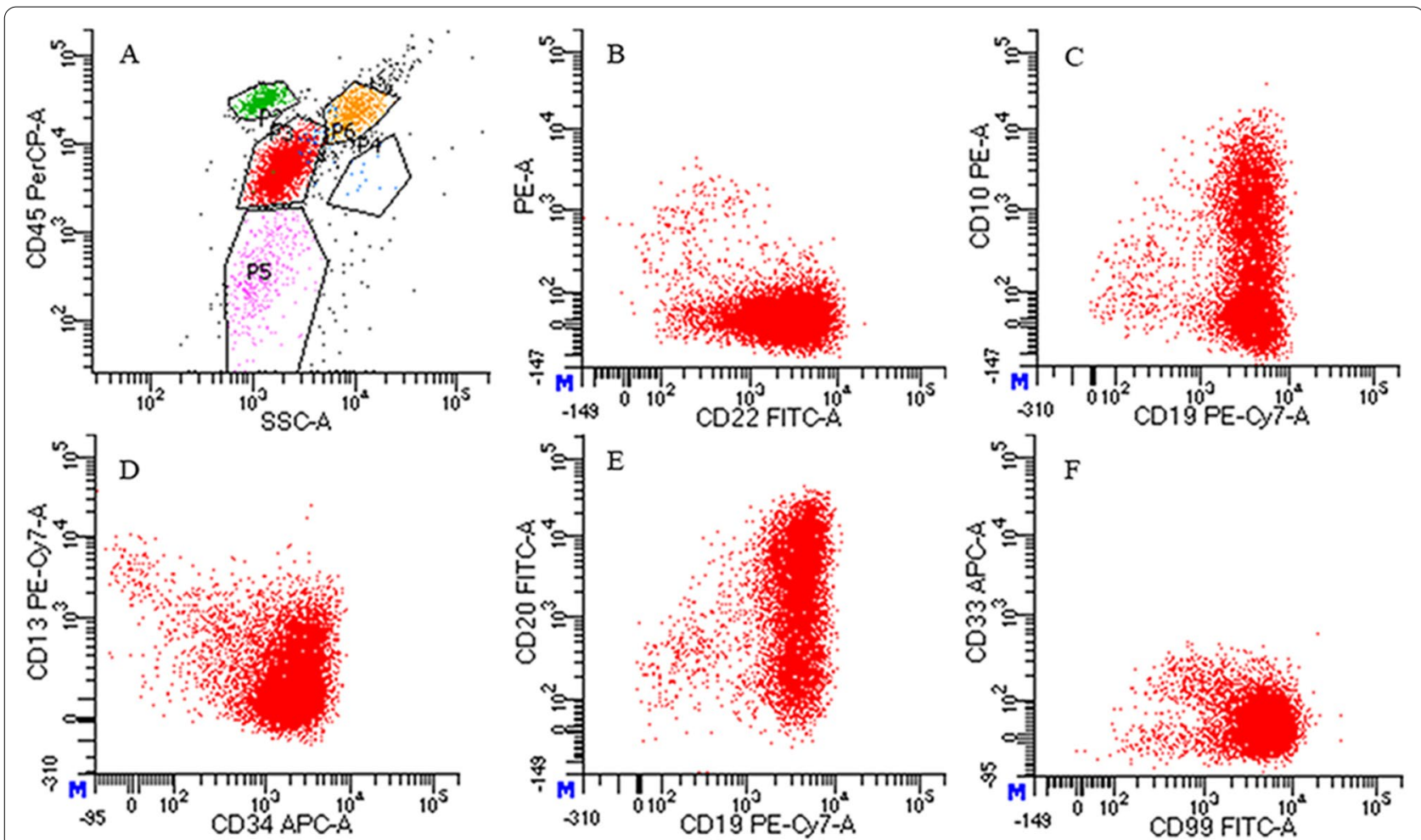

Fig. 3 The results of flow cytometry. The flow cytometry results indicated acute lymphocytic leukemia. A The proportion of primary cells was $68.45 \%$ according to the SSC/CD45 gating. B The CD22 fraction is shown as 22.8\%. C CD19 was positive, with a proportion of $81.1 \%$, and CD10 was positive, with a proportion of 20.9\%. D CD34 was positive, with a proportion of 98.2\%, and CD13 was positive, with a proportion of 55.8\%. E CD20 was positive, with a proportion of $35.0 \%$. F CD33 was positive, with a percentage of $69.8 \%$

prognostic factor in ALL because CD10-negative lymphoblastic leukemia cells had lower cycling capacities and were resistant to apoptosis $[15,16]$. A cohort study in China investigating 111 pediatric patients with BCP-ALL revealed that leukemic cells from patients with ZNF384 fusions were more likely to be CD10-negative than those from other pediatric patients $(18.8 \%$ vs. $2.9 \%, P=0.02)$ (17). This finding may explain why children with ZNF384 rearrangements have unfavorable outcomes. The adolescent patient in our case had the distinct immunophenotype of CD10 negativity, and she developed disease relapse only eight months after she reached CR despite receiving consistent consolidation chemotherapy. She also had poor responses to multiple chemotherapeutic therapies, necessitating frequent adjustments to more effective and powerful regimens. Our report supplements the unfavorable outcomes of adolescent patients with ZNF384 rearrangements in the existing literature and supports the view that ZNF384 rearrangements are correlated with a poor prognosis. However, larger-scale multicenter studies are required to evaluate the exact prognosis of ZNF384 rearrangements, especially in children or adolescents.
In conclusion, we report a BCP-ALL case with a ZNF384 rearrangement. The adolescent patient had very rare renal involvement as the first manifestation. However, she showed a very typical immunophenotype of ZNF384-rearranged ALL with CD10 negativity and $\mathrm{CD} 13$ and CD33 positivity. She responded poorly to chemotherapy and developed a relapse shortly after achieving CR. Her bone marrow karyotype was normal, which may suggest that the ZNF384 rearrangementrelated abnormal karyotype was under the detection limit of conventional G-banding. This indicates that more sensitive methods, such as FISH, should be used. Owing to the low detection rate and variety of treatment protocols from institution to institution, the statistical significance of clinical outcomes in children or adolescents with ZNF384 rearrangements has not been analyzed thus far. However, it has been linked to an unfavorable prognosis in some small studies, but this still needs to be replicated on a larger scale to be confirmed. Furthermore, ZNF384 break-apart probes are not commonly included in screening leukemia translocations, which could cause a delay in diagnosis and treatment. Therefore, we suggest that when the rearrangement of ZNF384 is suspected 
based on its characteristic immunophenotype, it should be tested and confirmed as soon as possible.

\begin{abstract}
Abbreviations
ALL: Acute lymphocytic leukemia; BCP-ALL: B-cell precursor acute lymphoblastic leukemia; ZNF384: Zinc-finger protein 384; FISH: Fluorescence in situ hybridization; FCM: Flow cytometry; NGS: Next generation sequencing; RT-PCR: Reverse transcription-polymerase chain reaction; CR: Complete remission; CCR: Continued complete remission; TCCSG: The Tokyo Children's Cancer Study Group; EFS: Event-free survival; OS: Overall survival.
\end{abstract}

\section{Supplementary Information}

The online version contains supplementary material available at https://doi. org/10.1186/s13039-022-00583-4.

\section{Additional file 1.}

\section{Acknowledgements}

Not applicable.

\section{Authors' contributions}

$\mathrm{JM}$ contributed to the explanation of laboratory data and the revision of the manuscript. JG wrote the manuscript. BC was responsible for supervising the data analysis. All authors read and approved the final manuscript.

\section{Funding}

This work was supported by grants from Key Medical of Jiangsu Province (ZDXKB2016020) and Nanjing Health Science and Technology Development Special Foundation (YKK18262).

\section{Availability of data and materials}

All data generated or analysed during this study are included in this published article [and its supplementary information files].

\section{Declarations}

Ethics approval and consent to participate Not applicable.

\section{Consent for publication}

Written informed consent was obtained from the patient for publication of this case report and any accompanying images. A copy of the written consent is available for review by the Editor-in-Chief of this journal.

\section{Competing interests}

The authors declare that they have no competing interests.

Received: 27 September 2021 Accepted: 28 January 2022

Published online: 14 February 2022

\section{References}

1. National Cancer Institute. SEER Cancer Statistics Review, 1975-2020 [Available from: https://seer.cancer.gov/statfacts/html/alyl.html.

2. Gurney JG, Severson RK, Davis S, Robison LL. Incidence of cancer in children in the United States. Sex-, race-, and 1-year age-specific rates by histologic type. Cancer. 1995;75(8):2186-95.

3. Nakamoto T, Yamagata T, Sakai R, Ogawa S, Honda H, Ueno H, et al. ClZ, a zinc finger protein that interacts with p130(cas) and activates the expression of matrix metalloproteinases. Mol Cell Biol. 2000;20(5):1649-58.

4. Jing Y, Li YF, Wan H, Liu DH. Detection of EP300-ZNF384 fusion in patients with acute lymphoblastic leukemia using RNA fusion gene panel sequencing. Ann Hematol. 2020;99(11):2611-7.
5. Hirabayashi S, Ohki K, Nakabayashi K, Ichikawa H, Momozawa Y, Okamura K, et al. ZNF384-related fusion genes define a subgroup of childhood B-cell precursor acute lymphoblastic leukemia with a characteristic immunotype. Haematologica. 2017;102(1):118-29.

6. Gocho Y, Kiyokawa N, Ichikawa H, Nakabayashi K, Osumi T, Ishibashi T, et al. A novel recurrent EP300-ZNF384 gene fusion in B-cell precursor acute lymphoblastic leukemia. Leukemia. 2015;29(12):2445-8.

7. Lilljebjörn H, Fioretos T. New oncogenic subtypes in pediatric B-cell precursor acute lymphoblastic leukemia. Blood. 2017;130(12):1395-401.

8. Alexander TB, Gu Z, lacobucci I, Dickerson K, Choi JK, Xu B, et al. The genetic basis and cell of origin of mixed phenotype acute leukaemia. Nature. 2018;562(7727):373-9.

9. Ma X, Edmonson M, Yergeau D, Muzny DM, Hampton OA, Rusch M, et al. Rise and fall of subclones from diagnosis to relapse in pediatric B-acute lymphoblastic leukaemia. Nat Commun. 2015;6:6604.

10. Yasuda T, Tsuzuki S, Kawazu M, Hayakawa F, Kojima S, Ueno T, et al. Recurrent DUX4 fusions in B cell acute lymphoblastic leukemia of adolescents and young adults. Nat Genet. 2016:48(5):569-74

11. Yaguchi A, Ishibashi T, Terada K, Ueno-Yokohata H, Saito Y, Fujimura J, et al. EP300-ZNF384 fusion gene product up-regulates GATA3 gene expression and induces hematopoietic stem cell gene expression signature in B-cell precursor acute lymphoblastic leukemia cells. Int J Hematol. 2017;106(2):269-81.

12. Hirabayashi S, Butler ER, Ohki K, Kiyokawa N, Bergmann AK, Möricke A, et al. Clinical characteristics and outcomes of B-ALL with ZNF384 rearrangements: a retrospective analysis by the Ponte di Legno Childhood ALL Working Group. Leukemia. 2021;35(11):3272-7.

13. Shago M, Abla O, Hitzler J, Weitzman S, Abdelhaleem M. Frequency and outcome of pediatric acute lymphoblastic leukemia with ZNF384 gene rearrangements including a novel translocation resulting in an ARID1B/ ZNF384 gene fusion. Pediatr Blood Cancer. 2016;63(11):1915-21.

14. Nishimura A, Hasegawa D, Hirabayashi S, Kanabuchi S, Yamamoto K, Aiga S, et al. Very late relapse cases of TCF3-ZNF384-positive acute lymphoblastic leukemia. Pediatr Blood Cancer. 2019;66(11):e27891.

15. Reaman GH, Sposto R, Sensel MG, Lange BJ, Feusner JH, Heerema NA, et al. Treatment outcome and prognostic factors for infants with acute lymphoblastic leukemia treated on two consecutive trials of the Children's Cancer Group. J Clin Oncol. 1999;17(2):445-55.

16. Cutrona G, Tasso P, Dono M, Roncella S, Ulivi M, Carpaneto EM, et al. CD10 is a marker for cycling cells with propensity to apoptosis in childhood ALL. Br J Cancer. 2002;86(11):1776-85.

17. Liu YF, Wang BY, Zhang WN, Huang JY, Li BS, Zhang M, et al. Genomic profiling of adult and pediatric B-cell acute lymphoblastic leukemia. EBioMedicine. 2016:8:173-83.

\section{Publisher's Note}

Springer Nature remains neutral with regard to jurisdictional claims in published maps and institutional affiliations.
Ready to submit your research? Choose BMC and benefit from:

- fast, convenient online submission

- thorough peer review by experienced researchers in your field

- rapid publication on acceptance

- support for research data, including large and complex data types

- gold Open Access which fosters wider collaboration and increased citations

- maximum visibility for your research: over 100M website views per year

At BMC, research is always in progress.

Learn more biomedcentral.com/submissions 